\title{
Fabrication and optical properties of periodical structures based on a water-developable and tunable $\mathrm{La}_{0.7} \mathrm{Sr}_{0.3} \mathrm{MnO}_{3}$ resist $\uparrow$
}

\author{
Ming-Chung Wu, ${ }^{a}$ Chih-Min Chuang, ${ }^{a}$ Yang-Fang Chen ${ }^{* b}$ and Wei-Fang $\mathrm{Su}^{* a}$
}

\author{
Received 24th September 2007, Accepted 11th December 2007 \\ First published as an Advance Article on the web 8th January 2008 \\ DOI: 10.1039/b714653a
}

\begin{abstract}
We have fabricated a unique periodic structure with different optical properties in the visible range using a water-developable $\mathrm{La}_{0.7} \mathrm{Sr}_{0.3} \mathrm{MnO}_{3}$ electron beam resist. The $\mathrm{La}_{0.7} \mathrm{Sr}_{0.3} \mathrm{MnO}_{3}$ resist can be developed under a nontoxic and environmentally friendly manner using pure water. Additionally, the resist is capable of exhibiting both positive and negative resist behavior depending on the electron beam dosage. Thus, these special characteristics were used to fabricate and tune periodic structure thin films having controlled optical reflectance properties in the wavelength range of $300 \mathrm{~nm}$ to $800 \mathrm{~nm}$ with one fixed design electron beam pattern (i.e. without changing the geometries and lattice constants of the periodical structures) by simply changing the electron beam dosage only.
\end{abstract}

\section{Introduction}

Recently, considerable interest has been focused on light modulation using periodic structures such as photonic crystals. ${ }^{1-6}$ Yablonovitch ${ }^{7}$ and $\mathrm{John}^{8}$ first described photonic crystals in 1987. The structures exhibit photonic band gaps, similar to the electronic band gaps of materials. Therefore, one can control the propagation of light by restricting its allowable directions at certain frequencies or localizing light in specific areas. The technology is expected to have numerous applications in optical communication. Many methods have been developed to fabricate photonic crystals, such as multilayer lithography, ${ }^{9}$ multibeam holographic lithography, ${ }^{10}$ self-assembly, ${ }^{11-13}$ step and flash imprint lithography, ${ }^{14}$ electrochemical deposition, ${ }^{15}$ etc. Among all the methods, electron beam lithography ${ }^{16-17}$ has become the most common method to fabricate 2D photonic crystals and nanoscale patterns, because of its ability to construct high precision structures.

The formation of photonic band gaps, which can be used to guide the propagation of light as well as to enhance spontaneous emission, is due to periodically varying refractive indices. Thus, an electron beam patternable metal oxide with high refractive index will be useful for this process. The nanostructure fabrication of metal oxide materials generally starts with selective etching or template growth on the pre-patterned resist and then lift-off. While these approaches lack the ability to control the construction of the nanostructure accurately, using an electron beam to generate exact nanopatterned metal oxides like $\mathrm{ZnO}, \mathrm{TiO}_{2}$, etc. ${ }^{18,19}$ can be much easier. The methods and materials mentioned above all generate negative patterns. There is a zwitter resist, ${ }^{20,21}$ which refers to a resist that can exhibit both positive and negative properties depending on the applied

${ }^{a}$ Department of Materials Science and Engineering, National Taiwan University, No. 1, Sec. 4, Roosevelt Road, Taipei, Taiwan, 106-17, ROC. E-mail: suwf@ntu.edu.tw; Fax: +886-2-33664078; Tel: +886-2-33664078 ${ }^{b}$ Department of Physics, National Taiwan University, No. 1, Sec. 4, Roosevelt Road, Taipei, Taiwan, 106-17, ROC. E-mail: yfchen@phys. ntu.edu.tw; Tel: $+886-2-33665125$

$\uparrow$ Electronic supplementary information (ESI) available: Absorption data. See DOI: 10.1039/b714653a electron beam dosage. The resist possesses both positive and negative patterning capabilities and will benefit the direct writing technology of the electron beam. In addition, the solvents used in the resist developing process are often volatile and toxic and thus contribute to health hazards and environmental pollution. The solvents even increase the cost of lithography processing. Many studies are directed towards building multifunctional resists with precise nanostructures and at the same time reducing the process hazards and pollution.

Herein we illustrate one unique feature of a patterned $\mathrm{La}_{0.7} \mathrm{Sr}_{0.3} \mathrm{MnO}_{3}$ film which is able to exhibit both positive and negative resist characteristics. This unique feature then can be used to control the optical reflectance properties of the film within one fixed design pattern without changing the lattice constant, shape and size of the pattern. Our approach provides a simple and convenient alternative for the fabrication of periodical structures with different optical properties in the visible range using a water-developable $\mathrm{La}_{0.7} \mathrm{Sr}_{0.3} \mathrm{MnO}_{3}$ resist.

\section{Experimental}

The electron beam resist was prepared by dissolving $8.58 \mathrm{wt} \%$ of lanthanum nitrate $\left(\mathrm{La}\left(\mathrm{NO}_{3}\right)_{3} \cdot 6 \mathrm{H}_{2} \mathrm{O}\right.$, Acros, $\left.98 \%\right)$, strontium nitrate $\left(\mathrm{Sr}\left(\mathrm{NO}_{3}\right)_{2}\right.$, Fluka, $\left.98 \%\right)$, manganese nitrate $\left(\mathrm{Mn}\left(\mathrm{NO}_{3}\right)_{2}\right.$. $4 \mathrm{H}_{2} \mathrm{O}$, Fluka, 97\%) and $1.75 \mathrm{wt} \%$ polyvinyl alcohol (PVA, Acros, $88 \%, 22000 \mathrm{~g} \mathrm{~mol}^{-1}$ ) in water with a molar ratio of $\mathrm{La}: \mathrm{Sr}: \mathrm{Mn}=$ $0.7: 0.3: 1.0$. The precursor solution was stirred for $48 \mathrm{~h}$ at $50^{\circ} \mathrm{C}$. After filtering the precursor solution with syringe filters $(0.22 \mu \mathrm{m})$, the precursor solution was spin coated at $3000 \mathrm{rpm}$ for $90 \mathrm{~s}$ to give a nominal thickness of about $200 \mathrm{~nm}$, and the resist film appeared to be very smooth with a root-mean-square roughness of $1.02 \mathrm{~nm}$. The thickness of the water-developable $\mathrm{La}_{0.7} \mathrm{Sr}_{0.3} \mathrm{MnO}_{3}$ resist coated on the substrate (indium tin oxide conductive glass or silicon wafer) was measured directly by using an alpha-stepper (Veeco, Tektak3). High-resolution nanolithography was performed by using a Hitachi ELS-7500EX machine to write the specific patterns across a $150 \times 150 \mu \mathrm{m}$ field with a $2.5 \mathrm{~nm}$ beam step size. The electron beam writer was operated at $50 \mathrm{kV}$ with a probe current of $1.0 \mathrm{nA}$. A sample containing a $3 \times 3$ array field 
was irradiated with a starting dose time of $1 \mu \mathrm{s}$, then with additional dose increments of $0.01,0.1$ and $1 \mu$ s per field. Deionized water was used to develop the $\mathrm{La}_{0.7} \mathrm{Sr}_{0.3} \mathrm{MnO}_{3}$ resist at $25^{\circ} \mathrm{C}$ for 30 s. The refractive indices of the $\mathrm{La}_{0.7} \mathrm{Sr}_{0.3} \mathrm{MnO}_{3}$ thin films sintered under different conditions were evaluated using a spectral microreflectometer (Mission Peaks Optics, MP100-M) equipped with an optical microscope. The patterned $\mathrm{La}_{0.7} \mathrm{Sr}_{0.3} \mathrm{MnO}_{3}$ samples were measured by atomic force microscopy (AFM, Digital Instruments, Dimension-3100 Multimode) for surface morphology and roughness, and the microstructures of the samples were studied by both field-emission scanning electron microscopy (FE-SEM, Elionix, ERA-8800FE, Japan) and confocal microscopy. The confocal microscopy images of the patterned $\mathrm{La}_{0.7} \mathrm{Sr}_{0.3} \mathrm{MnO}_{3}$ samples were measured by confocal fluorescence microscopy. An argon ion laser (wavelength $488 \mathrm{~nm}$ ) was used to excite the patterned $\mathrm{La}_{0.7} \mathrm{Sr}_{0.3} \mathrm{MnO}_{3}$ samples through a $100 \times$ Nikon plane objective (numerical aperture $\sim 0.9$ ). A single photon counting photomultiplier tube (PMT) detector was used to detect the intensity of the reflected laser light. The optical properties of the reflectance of the patterned $\mathrm{La}_{0.7} \mathrm{Sr}_{0.3} \mathrm{MnO}_{3}$ samples were studied using the transmittance mode of a spectral microreflectometer (Mission Peaks Optics, MP100-M) equipped with an optical microscope. Unpolarized light was focused on the composite film under the glass substrate at a spot size $<30 \mu \mathrm{m}$ to measure the interference between the incident and reflected light with wavelengths ranging from ultraviolet to near infrared (250-1000 nm).

\section{Results and discussion}

In our previous report ${ }^{22}$ we have demonstrated that a water developable $\mathrm{La}_{0.7} \mathrm{Sr}_{0.3} \mathrm{MnO}_{3}$ electron beam resist exhibits dual negative patterning and positive patterning functions depending on the electron beam dosage. Fig. 1(a)-(d) are atomic force microscope images of $\mathrm{La}_{0.7} \mathrm{Sr}_{0.3} \mathrm{MnO}_{3}$ patterns on silicon wafer substrates fabricated using different electron beam dosages. Fig. 1(a) is a negative pattern obtained at a low electron beam dose of $3.2 \mathrm{mC} \mathrm{cm}^{-2}$. Fig. 1(b) is a positive pattern obtained at a medium electron beam dose of $32.0 \mathrm{mC} \mathrm{cm}^{-2}$. At a high electron dose of $233.6 \mathrm{mC} \mathrm{cm}^{-2}$, we obtained a negative pattern again. Although the dose of the exposure of this $\mathrm{La}_{0.7} \mathrm{Sr}_{0.3} \mathrm{MnO}_{3}$ resist is very high in comparison to most organic resists, the $\mathrm{La}_{0.7} \mathrm{Sr}_{0.3} \mathrm{MnO}_{3}$ resist generates active electromagnetic functional nanopatterns in one step whereas the use of organic resists to generate $\mathrm{La}_{0.7} \mathrm{Sr}_{0.3} \mathrm{MnO}_{3}$ nanopatterns involves three steps: (1) fabricate nanopatterns from organic resist, (2) deposit $\mathrm{La}_{0.7} \mathrm{Sr}_{0.3} \mathrm{MnO}_{3}$ on the nanopatterns, and (3) remove organic resist. The use of $\mathrm{La}_{0.7} \mathrm{Sr}_{0.3} \mathrm{MnO}_{3}$ will not only reduce the process steps but also use no environmentally harmful organic chemicals as compared with the organic resist.

We observed a very unique and interesting pattern, featuring solid rod center-hollow ring-solid outer, that is 3 patterns of negative-positive-negative, at a medium-high electron beam dose of $134.4 \mathrm{mC} \mathrm{cm}^{-2}$. How does this unique pattern occur?
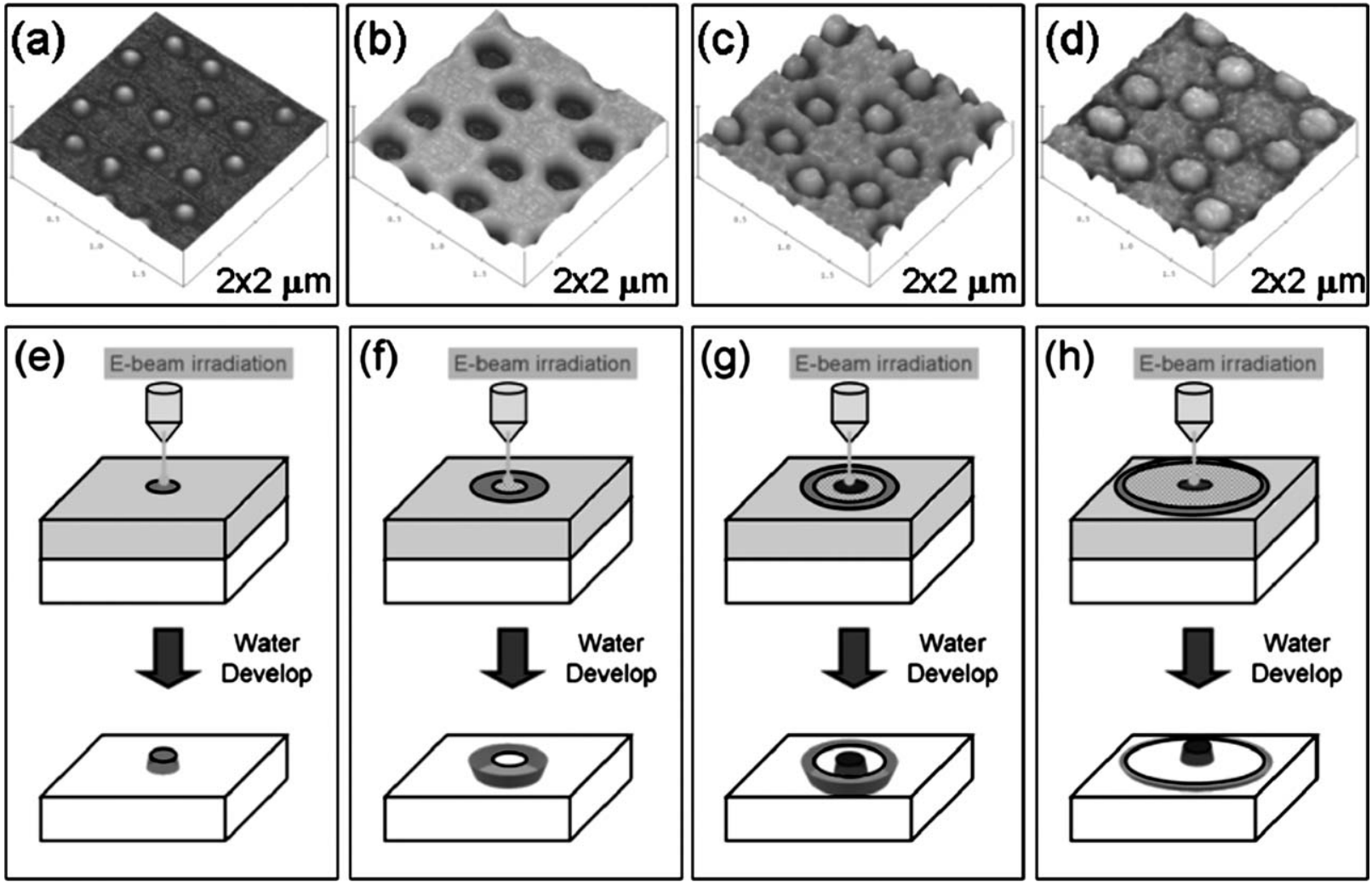

Fig. 1 Atomic force microscope (AFM) images of the $\mathrm{La}_{0.7} \mathrm{Sr}_{0.3} \mathrm{MnO}_{3}$ patterns on silicon wafer substrates with different electron beam dosages. (a) Negative $\mathrm{La}_{0.7} \mathrm{Sr}_{0.3} \mathrm{MnO}_{3}$ periodical structure with low dosage $\left(3.2 \mathrm{mC} \mathrm{cm}{ }^{-2}\right.$ ) electron beam irradiation. (b) Positive $\mathrm{La}_{0.7} \mathrm{Sr}_{0.3} \mathrm{MnO}_{3}$ periodical structure with medium dosage ( $32.0 \mathrm{mC} \mathrm{cm}{ }^{-2}$ ) electron beam irradiation. (c) Special unique $\mathrm{La}_{0.7} \mathrm{Sr}_{0.3} \mathrm{MnO}_{3}$ periodical structure with medium-high dosage (134.4 $\mathrm{mC} \mathrm{cm}{ }^{-2}$ ) electron beam irradiation. (d) Negative $\mathrm{La}_{0.7} \mathrm{Sr}_{0.3} \mathrm{MnO}_{3}$ periodical structure with high dosage $\left(233.6 \mathrm{mC} \mathrm{cm}^{-2}\right)$ electron beam irradiation. The schematic sketches of pattern formation mechanisms of $\mathrm{La}_{0.7} \mathrm{Sr}_{0.3} \mathrm{MnO}_{3}$ (e),(f),(g),(h) correspond to AFM images (a),(b),(c),(d) respectively. 
We have illustrated the formation mechanisms of the patterns in the lower row of Fig. 1(e)-(f).

We have previously confirmed the formation of the $\mathrm{La}_{0.7} \mathrm{Sr}_{0.3} \mathrm{MnO}_{3}$ resist pattern is due to the heat from the electron beam exposure. At the low electron dose, only the exposed area is affected by the crosslinking of the PVA (Fig. 1e) and results in a negative pattern of a regular honeycomb array of $\mathrm{La}_{0.7} \mathrm{Sr}_{0.3} \mathrm{MnO}_{3}$ pillars (Fig. 1a). At the medium dose $(28.8 \mathrm{mC}$ $\mathrm{cm}^{-2}$ ), the nitrate salt components of the $\mathrm{La}_{0.7} \mathrm{Sr}_{0.3} \mathrm{MnO}_{3}$ resist react with the PVA at the exposure area (Fig. 1f). An explosive reaction occurs, so-called auto-ignition, ${ }^{23-26}$ and then the resist structure will change to form loose particles that can be easily removed by water to form a positive pattern of a regular honeycomb array (Fig. 1b). At the medium-high dose, the centre part of the exposed area receives direct intense electron beam exposure (Fig. 1g) so a negative solid rod pattern is obtained (Fig. 1c). The area next to the center rod is exposed to the heat associated with the electron beam energy propagated to the neighbourhood which is equivalent to the medium dose (Fig. 1g). A hollow ring pattern is formed due to the positive resist behaviour at this energy level (Fig. 1c). The area further from the center is expected to receive a low electron beam dosage (Fig. 1g) which exhibits negative resist behaviour with a solid outer area formation (Fig. 1c). Finally, at the high dose, the area exposed to the electron beam (Fig. 1h) shows a negative pattern with the formation of crystalline $\mathrm{La}_{0.7} \mathrm{Sr}_{0.3} \mathrm{MnO}_{3}$ (Fig. 1d). At or above this electron beam thermal energy threshold, the pure phase of $\mathrm{La}_{0.7} \mathrm{Sr}_{0.3} \mathrm{MnO}_{3}$ formed as a solid pattern, the neighbouring loose $\mathrm{La}_{0.7} \mathrm{Sr}_{0.3} \mathrm{MnO}_{3}$ particles can be removed by water. Our $\mathrm{La}_{0.7} \mathrm{Sr}_{0.3} \mathrm{MnO}_{3}$ resist exhibits zwitter resist characteristics, so the height or depth in different patterns will varying with electron dose. The height of the regular honeycomb array of $\mathrm{La}_{0.7} \mathrm{Sr}_{0.3} \mathrm{MnO}_{3}$ pillars (Fig. 1a) is $15 \mathrm{~nm}$, and the depth of the positive pattern of the regular honeycomb array is $60 \mathrm{~nm}$ (Fig. 1b), Finally, the height of the regular honeycomb array of $\mathrm{La}_{0.7} \mathrm{Sr}_{0.3} \mathrm{MnO}_{3}$ pillars (Fig. 1d) is $75 \mathrm{~nm}$.

The refractive index of the electron beam exposed $\mathrm{La}_{0.7} \mathrm{Sr}_{0.3} \mathrm{MnO}_{3}$ resist is one of the key properties that need to be measured to understand the optical properties of patterned $\mathrm{La}_{0.7} \mathrm{Sr}_{0.3} \mathrm{MnO}_{3}$. However, the electron beam patterned $\mathrm{La}_{0.7} \mathrm{Sr}_{0.3} \mathrm{MnO}_{3}$ sample is too small to be measured accurately using conventional ellipsometer or prism coupler methods. We knew from previous work ${ }^{22}$ that the formation of $\mathrm{La}_{0.7} \mathrm{Sr}_{0.3} \mathrm{MnO}_{3}$ patterns is due to the thermal reactions of the components in the resist. Therefore, we measured the refractive indices of $\mathrm{La}_{0.7} \mathrm{Sr}_{0.3} \mathrm{MnO}_{3}$ samples prepared by thermal sintering with an equivalent energy to the electron beam energy. Table 1 summaries

Table 1 Summary of refractive indexes of $\mathrm{La}_{0.7} \mathrm{Sr}_{0.3} \mathrm{MnO}_{3}$ samples prepared under different sintering conditions

\begin{tabular}{llll}
\hline $\begin{array}{l}\text { Sample of } \\
\mathrm{La}_{0.7} \mathrm{Sr}_{0.3} \mathrm{MnO}_{3} \\
\text { thin film }\end{array}$ & $\begin{array}{l}\text { Sintering } \\
\text { conditions }\end{array}$ & $\begin{array}{l}\text { Equivalent to the } \\
\text { electron beam } \\
\text { dosage/mC cm }\end{array}$ & $\begin{array}{l}\text { Refractive } \\
\text { index }\end{array}$ \\
\hline $\mathrm{A}$ & - & 0.0 & $\sim 2.00$ \\
$\mathrm{~B}$ & $150^{\circ} \mathrm{C}, 0.5 \mathrm{~h}$ & $1.5-4.0$ & $\sim 2.05$ \\
$\mathrm{C}$ & $250^{\circ} \mathrm{C}, 0.5 \mathrm{~h}$ & $15.0-35.0$ & $\sim 2.05$ \\
$\mathrm{D}$ & $300^{\circ} \mathrm{C}, 0.5 \mathrm{~h}$ & $35.0-120.0$ & $\sim 2.30$ \\
$\mathrm{E}$ & $300^{\circ} \mathrm{C}, 30.0 \mathrm{~h}$ & $120.0-300.0$ & $\sim 2.38$ \\
\hline
\end{tabular}

the refractive indices of $\mathrm{La}_{0.7} \mathrm{Sr}_{0.3} \mathrm{MnO}_{3}$ thin film samples prepared under different sintering conditions. The negative pattern obtained at low electron beam dose has a refractive index of 2.05 whereas the negative pattern obtained at high electron beam dose has a higher refractive index of 2.38. The medium electron dose sample should have a refractive index of 2.05 because the exposed area is removed and the remaining area is a mixture of PVA and $\mathrm{La}_{0.7} \mathrm{Sr}_{0.3} \mathrm{MnO}_{3}$ precursor salts which is the same as the low electron dose irradiated sample. Thus, we can change the refractive index of the resist by varying the electron beam dosage.

We have used both scanning electron microscopy and confocal microscopy to further study the morphology of the electron beam irradiated resist as shown in Fig 2a, c, e, g and Fig. 2b,
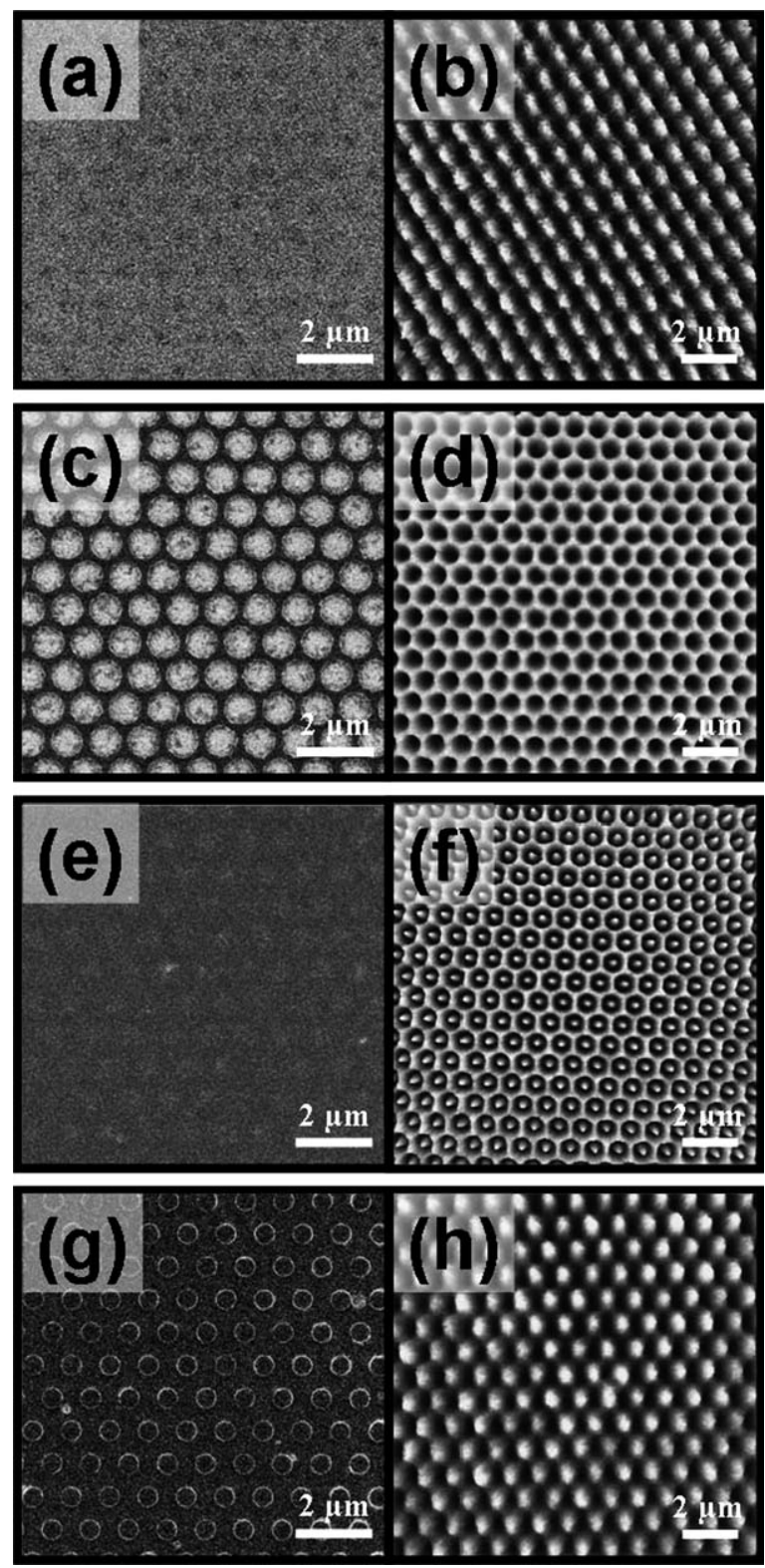

Fig. 2 Scanning electron microscopy photographs (a, c, e, and g) and confocal microscopy images (b, d, f, and $\mathrm{h}$ ) of $\mathrm{La}_{0.7} \mathrm{Sr}_{0.3} \mathrm{MnO}_{3}$ periodical structures fabricated from water developable resists at different electron beam dosages: (a,b) $2.2 \mathrm{mC} \mathrm{cm}^{-2}$, (c,d) $16.0 \mathrm{mC} \mathrm{cm}^{-2}$, (e,f) $80.0 \mathrm{mC} \mathrm{cm}^{-2}$, and $(\mathrm{g}, \mathrm{h}) 288.0 \mathrm{mC} \mathrm{cm}^{-2}$. 
$\mathrm{d}, \mathrm{f}, \mathrm{h}$ respectively. The confocal microscopy images are much clearer than the SEM images due to the small topographic contrast in SEM. The pattern was designed and set at $1000 \mathrm{~nm}$ lattice constant and $500 \mathrm{~nm}$ diameter. However, different characteristics of patterned $\mathrm{La}_{0.7} \mathrm{Sr}_{0.3} \mathrm{MnO}_{3}$ resists were obtained by only varying the electron beam dosage. The results are due to the different chemical reactions occurred in the resist as described above. Fig. 2a,b shows a periodical pillar array with 250 diameter and $1000 \mathrm{~nm}$ lattice constant, and the electron beam dosage in this step was $2.2 \mathrm{mC} \mathrm{cm}^{-2}$. A periodical hole array pattern with $800 \mathrm{~nm}$ diameter and $1000 \mathrm{~nm}$ lattice constant is shown in Fig. 2c,d, which was obtained at a low electron beam dose of $16.0 \mathrm{mC} \mathrm{cm}^{-2}$. However, we can observe a special pattern array with the same processing setting at $800 \mathrm{~nm}$ diameter and $1000 \mathrm{~nm}$ lattice constant at a medium-high electron beam dose of $80.0 \mathrm{mC} \mathrm{cm}^{-2}$ due to the heat transfer to the neighboring area (Fig. 2e,f). Finally, the $\mathrm{La}_{0.7} \mathrm{Sr}_{0.3} \mathrm{MnO}_{3}$ pillar array with $500 \mathrm{~nm}$ diameter and $1000 \mathrm{~nm}$ lattice constant was obtained at the high electron beam dose of $288.0 \mathrm{mC} \mathrm{cm}^{-2}$ as shown in Fig. 2g,h.

Traditionally, the optical properties of photonic crystals are modulated either by changing the radius with fixed lattice constant or by changing the lattice constant with fixed radius.
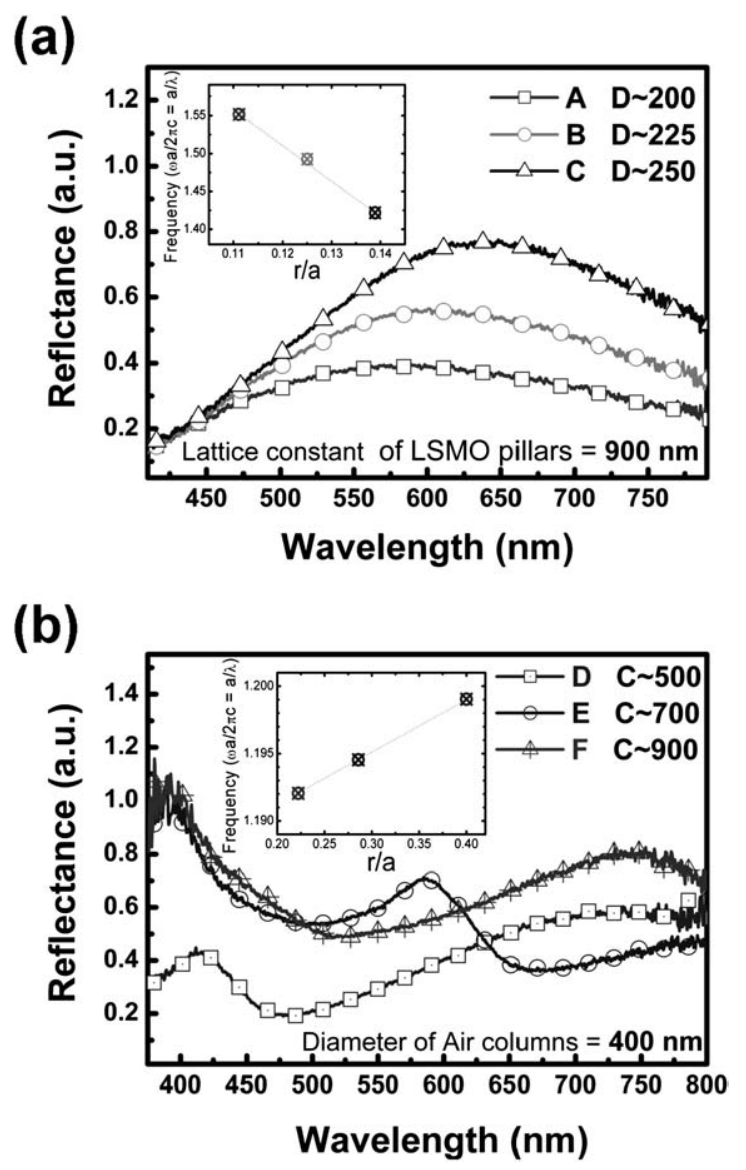

Fig. 3 Reflectance spectra changes of patterned $\mathrm{La}_{0.7} \mathrm{Sr}_{0.3} \mathrm{MnO}_{3}$ with different geometries. (a) The patterns with a fixed $900 \mathrm{~nm}$ lattice constant and different $\mathrm{La}_{0.7} \mathrm{Sr}_{0.3} \mathrm{MnO}_{3}$ pillar diameters: 200, 225, and $250 \mathrm{~nm}$. (b) The patterns with a fixed $400 \mathrm{~nm}$ air hole diameter and different lattice constants: 500, 700, and $900 \mathrm{~nm}$. Inserts are the plots of frequency versus r/a.
Therefore, we fabricated two types of $\mathrm{La}_{0.7} \mathrm{Sr}_{0.3} \mathrm{MnO}_{3}$ 2D photonic crystals on silicon wafers. One was a pillar structure fabricated at low electron dose and the other was an air column structure fabricated at medium electron dose. They have the size of about $50 \times 50 \mu \mathrm{m}$. For the pillar structure, we studied the reflectance spectra of $\mathrm{La}_{0.7} \mathrm{Sr}_{0.3} \mathrm{MnO}_{3}$ photonic crystals with fixed $900 \mathrm{~nm}$ lattice constant and different $\mathrm{La}_{0.7} \mathrm{Sr}_{0.3} \mathrm{MnO}_{3}$ pillar diameters of 200,225 , and $250 \mathrm{~nm}$ respectively. The results are shown in Fig. 3a, the samples were prepared using low dose electron beams, so their refractive indices (samples A, B and C) are about 2.05. Each reflectance curve of the samples was obtained by a spectral microreflectometer equipped with an optical microscope. The reflectance spectra show a red shift of $\lambda_{\max }$ from 580 $\mathrm{nm}$, to $603 \mathrm{~nm}$ and $633 \mathrm{~nm}$ upon increasing the pillar diameter from $200 \mathrm{~nm}$ to $225 \mathrm{~nm}$ and $250 \mathrm{~nm}$ respectively. Therefore, the reflectance spectra of the $2 \mathrm{D} \mathrm{La}_{0.7} \mathrm{Sr}_{0.3} \mathrm{MnO}_{3}$ photonic crystals can be adjusted by varying the geometric shape or filling ratio. The insert of Fig. 3a shows the relationship of the frequency $(a / \lambda)$ versus r/a ratio for the samples, which has a negative slope. The result is consistent with the theoretical prediction. ${ }^{27}$

We used the Maxwell equation to validate the shifting trend of the reflectance of the patterned $\mathrm{La}_{0.7} \mathrm{Sr}_{0.3} \mathrm{MnO}_{3}$ samples. By considering the light propagation in the $x-y$ plane within the triangular lattice of dielectric columns and the refractive index of the $\mathrm{La}_{0.7} \mathrm{Sr}_{0.3} \mathrm{MnO}_{3}$ resist film, the band structures for photonic crystals of $\mathrm{La}_{0.7} \mathrm{Sr}_{0.3} \mathrm{MnO}_{3}$ columns with different $r / a$ values were calculated by using the BANDSOLVE program (RSOFT Design Group, Inc). This calculation was performed using the plane-wave expansion method and index guiding in the third dimension. ${ }^{28}$ The refractive index of $\mathrm{La}_{0.7} \mathrm{Sr}_{0.3} \mathrm{MnO}_{3}$ pillars irradiated at $1.5-4.0 \mathrm{mC} \mathrm{cm}^{-2}$ is about 2.05 . Hence, the refractive index of 2.05 for $\mathrm{La}_{0.7} \mathrm{Sr}_{0.3} \mathrm{MnO}_{3}$ pillar samples and a domain size of $7 \times 11$ unit cells were used in this calculation. In the $\mathrm{La}_{0.7} \mathrm{Sr}_{0.3} \mathrm{MnO}_{3}$ pillar structures, the frequency of the gap is decreased as $r / a$ increases. This is expected, because the frequency is related to $1 / \sqrt{ } \varepsilon$, where $\varepsilon$ is the dielectric constant of the medium; when $r / a$ increases, the average dielectric constant of the medium increases and as a result the frequency increases.

Moreover, for the air column structure, the reflectance spectra of $\mathrm{La}_{0.7} \mathrm{Sr}_{0.3} \mathrm{MnO}_{3}$ photonic crystals with a fixed $400 \mathrm{~nm}$ air column diameter and different lattice constants: 500, 700, and $900 \mathrm{~nm}$ (Fig. 3b; samples D, E and F respectively) show a red shift from $417 \mathrm{~nm}$, to $586 \mathrm{~nm}$ and $755 \mathrm{~nm}$. Thus, the $\lambda_{\max }$ of the samples increase with increasing lattice constant. The insert in Fig. $3 b$ is the relationship of the frequency $(a / \lambda)$ versus r/a ratio, which is positive. The frequency of the gap increases as $r / a$ increases in the air column structures. This is also an expected feature, as explained above.

Interestingly, our dual function $\mathrm{La}_{0.7} \mathrm{Sr}_{0.3} \mathrm{MnO}_{3}$ resist exhibits additional unique characteristics that can modulate the optical properties of photonic crystals by changing the electron dose only with a fixed column diameter and lattice constant. Fig. 4 shows the reflection spectra of patterned $\mathrm{La}_{0.7} \mathrm{Sr}_{0.3} \mathrm{MnO}_{3}$ fabricated with different electron doses using a fixed design of 500 $\mathrm{nm}$ diameter and $1000 \mathrm{~nm}$ lattice constant. Each curve represents a sample obtained at a specific electron irradiation as shown on the right. The thickness of the $\mathrm{La}_{0.7} \mathrm{Sr}_{0.3} \mathrm{MnO}_{3}$ resist coated on the silicon wafer substrate was about $200 \mathrm{~nm}$. The sample size was $50 \times 50 \mu \mathrm{m}$. By increasing the electron beam irradiation, 


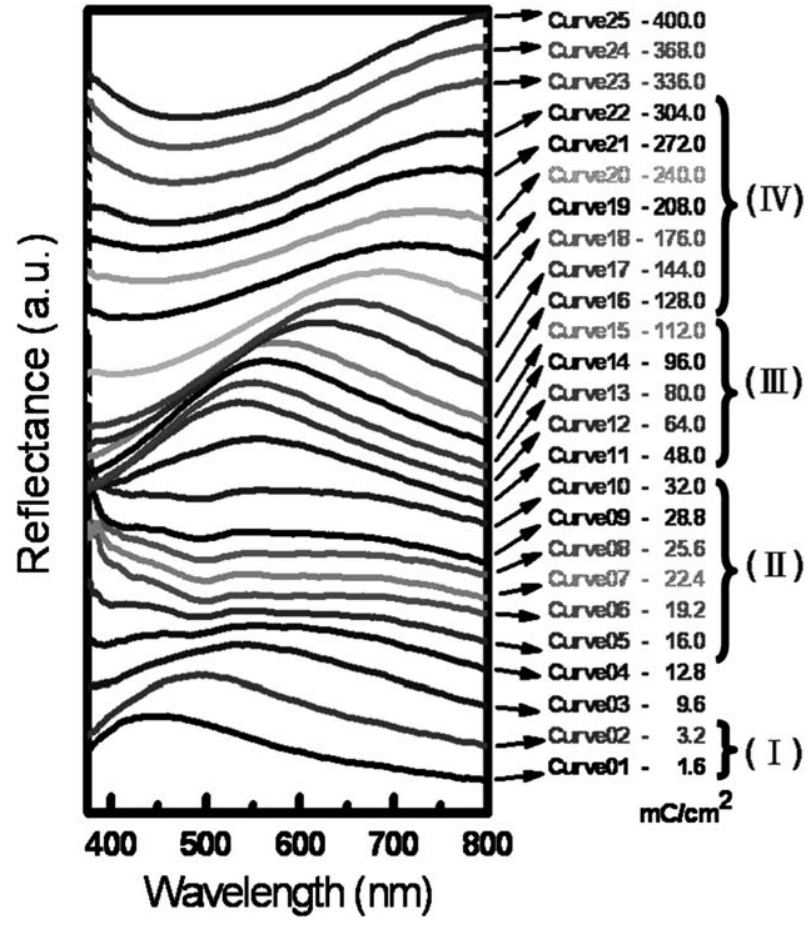

Fig. 4 Reflectance spectra of patterned $\mathrm{La}_{0.7} \mathrm{Sr}_{0.3} \mathrm{MnO}_{3}$ were changed by varying the electron beam dosage, and the design pattern is fixed at $1000 \mathrm{~nm}$ lattice constant and $500 \mathrm{~nm}$ diameter. Four ranges of electron beam dose were used. Low dose region (I): $1.6-3.2 \mathrm{mC} \mathrm{cm}^{-2}$, medium dose region (II): $12.8-32.0 \mathrm{mC} \mathrm{cm}{ }^{-2}$, medium-high dose region (III): $48.0-112.0 \mathrm{mC} \mathrm{cm}^{-2}$, and high dose region (IV): $128.0-304.0 \mathrm{mC} \mathrm{cm}^{-2}$.

we can obtain different geometries of patterned $\mathrm{La}_{0.7} \mathrm{Sr}_{0.3} \mathrm{MnO}_{3}$ that result in the changes in the reflectance spectra.

First of all, the negative patterned $\mathrm{La}_{0.7} \mathrm{Sr}_{0.3} \mathrm{MnO}_{3}$ irradiated at 1.6 and $3.2 \mathrm{mC} \mathrm{cm}^{-2}$ show $\lambda_{\max }$ at 445 and $480 \mathrm{~nm}$ (Curve 01 and Curve 02) respectively in the low dose region (I). When the positive patterned $\mathrm{La}_{0.7} \mathrm{Sr}_{0.3} \mathrm{MnO}_{3}$ was obtained by the increasing electron beam dosage in the range from 16.0 to 32.0 $\mathrm{mC} \mathrm{cm}^{-2}$, the reflectance spectra are shown in the medium dose region (II) (Curve 05-Curve 10). Nevertheless, the patterned $\mathrm{La}_{0.7} \mathrm{Sr}_{0.3} \mathrm{MnO}_{3}$ in the medium-high region (III) was transformed from a positive pattern to a negative pattern gradually, when the electron beam dosage was increased to the range of $48.0-112.0 \mathrm{mC} \mathrm{cm}^{-2}$. The $\lambda_{\max }$ of the reflectance peaks in the region (III) are blue shifted initially (Curve-11 to Curve12) and then they are red shifted (Curve 12-Curve 15). These behaviors are due to the changes of air column geometry as shown in Fig 2f. In other words, the depth of the air column in patterned $\mathrm{La}_{0.7} \mathrm{Sr}_{0.3} \mathrm{MnO}_{3}$ samples increases gradually from Curve 11 to Curve 12 due to the positive characteristics of the resist, then decreases gradually from Curve 12 to Curve 15 due to the gradual transformation of the negative characteristics of the resist. The results reveal that the $\mathrm{La}_{0.7} \mathrm{Sr}_{0.3} \mathrm{MnO}_{3}$ resist can easily modulate $2 \mathrm{D}$ photonic crystal reflectance spectra in the visible range by varying the $z$-axis using different electron beam dosages without changing the design pattern of lattice constant and the periodicity along the $x$ - and $y$-axes. Finally, the negative patterned $\mathrm{La}_{0.7} \mathrm{Sr}_{0.3} \mathrm{MnO}_{3}$ was obtained in region (IV) with the electron beam dosage range of $128.0-304.0 \mathrm{mC}$

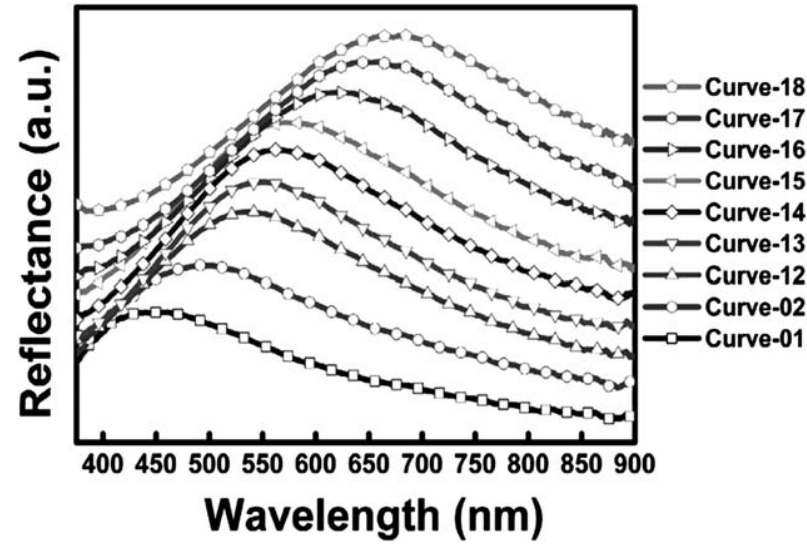

Fig. 5 Modulation of the reflectance spectra in the visible range using $\mathrm{La}_{0.7} \mathrm{Sr}_{0.3} \mathrm{MnO}_{3}$ periodical structures fabricated by varying the electron beam dosage at a fixed design pattern of $1000 \mathrm{~nm}$ lattice constant and $500 \mathrm{~nm}$ diameter.

$\mathrm{cm}^{-2}$, and their reflectance spectra are shown in Curve 16Curve 22. Therefore, we can easily use a water-developable $\mathrm{La}_{0.7} \mathrm{Sr}_{0.3} \mathrm{MnO}_{3}$ resist to fabricate periodical structures with different optical properties. This unique feature of the periodic structure of $\mathrm{La}_{0.7} \mathrm{Sr}_{0.3} \mathrm{MnO}_{3}$ is replotted in Fig. 5 for clear demonstration of visible light modulation.

\section{Conclusions}

In summary, we have created unique periodical structures using a water-developable $\mathrm{La}_{0.7} \mathrm{Sr}_{0.3} \mathrm{MnO}_{3}$ electron beam resist in an environmentally friendly, one-step method. Both positive and negative patterns are readily fabricated by only varying the electron beam dosage. Different reflectance spectra of patterned $\mathrm{La}_{0.7} \mathrm{Sr}_{0.3} \mathrm{MnO}_{3}$ in the visible light range can be obtained. We would like to stress that our study provides a simple and convenient approach for the fabrication of photonic crystals with adjustable optical properties, which may underpin many optoelectronic or magnetic applications awaiting future exploration.

\section{Acknowledgements}

Financial support from the National Science Council of Taiwan (NSC-96-2628-E-002-017-MY3 and NSC 95-3114-P-002-003MY3) is highly appreciated. The authors would also like to thank Prof. C. H. Kuan, Prof. C. W. Chen, Ms S. Chen and Mr Y. Y. Lin of National Taiwan University and Prof. K. C. Cheng of National Taipei University for helpful discussions, and $\mathrm{Mr} \mathrm{A}$. J. Su of University of Pittsburgh for editing the manuscript. The electron beam lithography was carried out using the Elinox's facility located at the National Taiwan University Center for Information and Electronics Technologies.

\section{References}

1 M. Geissler and Y. Xia, Adv. Mater., 2004, 16, 1249.

2 P. V. Braun, S. A. Rinne and F. García-Santamaría, Adv. Mater., 2006, 18, 2665.

3 J. Y. Cheng, C. A. Ross, H. I. Smith and E. L. Thomas, Adv. Mater., 2006, 18, 2505. 
4 B. Maune, M. Loncar, J. Witzens, M. Hochberg, T. B. Jones and Y. Qiu, Appl. Phys. Lett., 2004, 85, 360.

5 C. M. Chuang, W. B. Lu, W. F. Su, C. M. Lin and Y. F. Chen, J. Appl. Phys., 2005, 97, 096104.

6 Y. L. Chen, C. C. Chen, J. C. Jeng and Y. F. Chen, Appl. Phys. Lett., 2004, 85, 1259

7 E. Yablonovitch, Phys. Rev. Lett., 1987, 58, 2059.

8 S. John, Phys. Rev. Lett., 1987, 58, 2468.

9 S. Noda, MRS Bull., 2001, 26, 618.

10 J. Turberfield, MRS Bull., 2001, 26, 632.

11 S. H. Park and Y. N. Xia, Langmuir, 1999, 15, 266.

12 Q. Yan, Z. Zhou, X. S. Zhao and S. J. Chua, Adv. Mater., 2005, 17, 1917.

13 T. Nakamura, Y. Yamada and K. Yano, J. Mater. Chem., 2007, 17, 3726

14 K. Long, B. K. Keitz and C. G. Willson, J. Mater. Chem., 2007, 17, 3575.

15 Y. W. Su, C. S. Wu, C. C. Chen and C. D. Chen, Adv. Mater., 2005, 15, 49.

16 G. Subramania and S. Y. Lin, Appl. Phys. Lett., 2004, 85, 5037.

17 D. Yang, S. W. Chang and C. K. Ober, J. Mater. Chem., 2006, 16, 1693.

18 M. S. M. Saifullah, K. R. V. Subramanian, E. Tapley, D. Kang, M. E. Welland and M. Butler, Nano Lett., 2003, 3, 1587.
19 M. S. M. Saifullah, K. R. V. Subramanian, D. Kang, D. Anderson, W. T. S. Huck, G. A. C Jones and M. E. Welland, Adv. Mater., 2005, 17, 1757.

20 J. K. Chen, F. H. Ko, H. L. Chen and F. C. Chang, Jpn. J. Appl. Phys., 2003, 42, 3838

21 J. K. Chen, F. H. Ko and F. C. Chang, Adv. Funct. Mater., 2005, 15, 1147.

22 C. M. Chuang, M. C. Wu, Y. C. Huang, K. C. Cheng, C. F. Lin, Y. F. Chen and Y. F. Su, Nanotechnology, 2006, 17, 4399.

23 R. N. Basu, S. K. Pratihar, M. Saha and H. S. Maiti, Mater. Lett., 1997, 32, 217.

24 A. Chakraborty, P. S. Devi, S. Roy and H. S. Maiti, J. Mater. Res., 1994, 9, 986.

25 M. Gaudon, C. Laberty-Robert, F. Ansart, P. Stevens and A. Rousset, Solid State Sci., 2002, 4, 125.

26 S. A. Antony, K. S. Nagaraja, G. L. N. Reddy and O. M. Sreedharan, Mater. Lett., 2001, 51, 414.

27 J. D. Joannopoulos, R. D. Meade and J. N. Winn, Photonic Crystals: Molding the Flow of Light, Princeton Univ. Press, New Jersey, USA, 1995.

28 S. G. Johnson, S. Fan, P. R. Villeneuve and J. D. Joannopoulos, Phys. Rev. B, 1999, 60, 5751. 